\title{
Editorial
}

\section{The price of cumulative human activities in the Antarctic}

$\mathrm{O}$ ne of the main and most accessible logistic hubs for the Antarctic Peninsula is located on Fildes Peninsula in the south-western part of King George Island, South Shetland Islands. By Antarctic standards, this area hosts an extraordinarily high concentration of human activities, encompassing scientific research, logistics and tourism. At the same time, the area is characterized by relatively high biodiversity and hosts two Antarctic Specially Protected Areas as well as various historical values. Numerous legally binding conventions and non-binding provisions and (and in particular the Protocol on Environmental Protection to the Antarctic Treaty, which came into force in 1998), apply to those working on and travelling through the Fildes Peninsula, as they do to all individuals, operators and operations across Antarctica. However, as a result of the long human presence in this area and the cumulative effects of human activities, a wide range of negative local anthropogenic local impacts have been repeatedly and clearly reported. Some of the identified practical and procedural deficiencies underlying these are progressively being addressed, particularly in areas such as sewage treatment, fuel and waste management, dissemination of information and monitoring of non-native species. Nevertheless, there is a considerable way to go and the current momentum must not be lost. There are many ongoing, cumulative and serious challenges including, for example, the continuing practice of open waste burning without efforts to "reduce harmful emissions" or "to limit particulate deposition" As mandated in the Protocol, disposal of waste into the environment (littering) by both national operator staff and other visitors, the presence of non-native soils and house plant species within station buildings, deterioration and pollution through poor or no maintenance of present or past infrastructure, repeated minor or major oil spills, inconsistent levels of sewage treatment and control of effluent release, high levels of (unpermitted) human disturbance of animals even by station personnel and official delegations, nearshore and shoreline pollution from marine debris, and poor to no application of recommended biosecurity procedures aimed to prevent the introduction of non-native species. Most recently, in an article in Biology Letters, microplastics have been found in terrestrial springtails close to the shore on Fildes Peninsula. An area such as this hosting multiple national scientific, logistic and commercial operations would seem to be crying out for designation and international management as an Antarctic Specially Managed Area (ASMA). Two other ASMAs already exist in similarly intensively used parts of the South Shetland Islands subject to the activity of multiple operators, Admiralty Bay (also on King George Island) and Deception Island, providing clear models for what could be achieved on Fildes Peninsula. The management challenges experienced in Fildes Peninsula, exacerbated by a general lack of coordination and application of consistent approaches between different operators, have been highlighted frequently by the scientific community through SCAR and other fora, as well as receiving negative media attention. Perhaps surprisingly, this does not yet appear to have catalysed sufficiently serious engagement by the Parties to the ATCM, where decision making power lies. Notwithstanding the current hiatus on most activities caused by the COVID-19 pandemic, the need for effective action within the Antarctic Treaty System is becoming increasingly urgent. In recent years, a considerable increase in all types of human activities has been clear on Fildes Peninsula, most striking being expansion in national operator traffic and personnel, further growth of air-cruise tourist programs and sporting events, and the creation of at least one tourist camp for overnight stays, with none of the latter being able to exist without sometimes substantial collaboration with National Antarctic Programmes. These upwards trends are expected to resume as the current global crisis recedes. Clearly, strict, and consistent implementation of the existing legal requirements, guidelines and recommendations is vital for the future rational use and protection of Fildes Peninsula and its habitats and ecosystems. The question is already being openly asked of whether we are blindly, or even deliberately, acquiescing in the creation of an Antarctic sacrificial area in the name of servicing logistic and tourism needs, or are prepared to redouble efforts to convince all regional stakeholders to avoid further degradation of the local environment and engage energetically and constructively to achieve one of the founding principles of the Antarctic Treaty itself, to protect and preserve Antarctica and its ecosystems.

Peter Convey

\section{References}

Bergami, E, Rota, E, Caruso, T, Birarda, G, Vaccari, L. \& Corsi, I. 2020. Plastics everywhere: first evidence of polystyrene fragments inside the common Antarctic collembolan Cryptopygus antarcticus. Biology Letters, 16, 10.1098/rsbl.2020.0093. 